\title{
Recent progress on JET towards the ITER reference mode of operation at high density
}

\author{
J Ongena ${ }^{1,17}$, W Suttrop ${ }^{2}$, M Bécoulet ${ }^{3}$, G Cordey $^{4}$, P Dumortier ${ }^{1}$, \\ Th Eich $^{5}$, L C Ingesson ${ }^{6}$, S Jachmich ${ }^{1}$, P Lang ${ }^{5}$, A Loarte ${ }^{7}$, P Lomas ${ }^{4}$, \\ G P Maddison ${ }^{4}$, A Messiaen ${ }^{1}$, M F F Nave ${ }^{8}$, J Rapp ${ }^{5}$, G Saibene ${ }^{7}$, \\ R Sartori ${ }^{7}$, O Sauter ${ }^{9}$, J D Strachan ${ }^{10}$, B Unterberg ${ }^{5}$, M Valovic $^{4}$, \\ B Alper ${ }^{4}$, Ph Andrew ${ }^{4}$, Y Baranov ${ }^{4}$, J Brzozowski $^{10}$, J Bucalossi $^{3}$, \\ M Brix ${ }^{5}$, R Budny ${ }^{11}$, M Charlet ${ }^{4}$, I Coffey ${ }^{4}$, M De Baar ${ }^{6}$, P De Vries ${ }^{6}$, \\ C Gowers $^{4}$, N Hawkes ${ }^{4}$, M von Hellermann ${ }^{6}$, D L Hillis ${ }^{12}$, J Hogan ${ }^{12}$, \\ G L Jackson ${ }^{13}$, E Joffrin ${ }^{3}$, C Jupen ${ }^{10}$, A Kallenbach ${ }^{2}$, H R Koslowski ${ }^{5}$, \\ K D Lawson ${ }^{4}$, M Mantsinen ${ }^{4}$, G Matthews ${ }^{4}$, P Monier-Garbet ${ }^{3}$, \\ D McDonald ${ }^{4}$, F Milani ${ }^{4}$, M Murakami ${ }^{12,13}$, A Murari $^{14}$, R Neu $^{2}$,

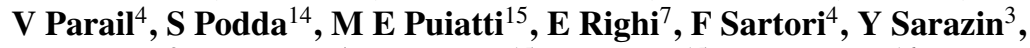 \\ A Staebler ${ }^{2}$, M Stamp $^{4}$, G Telesca $^{15}$, M Valisa ${ }^{15}$, B Weyssow ${ }^{16}$, \\ K-D Zastrow ${ }^{4}$ and EFDA-JET workprogramme contributors ${ }^{18}$
}

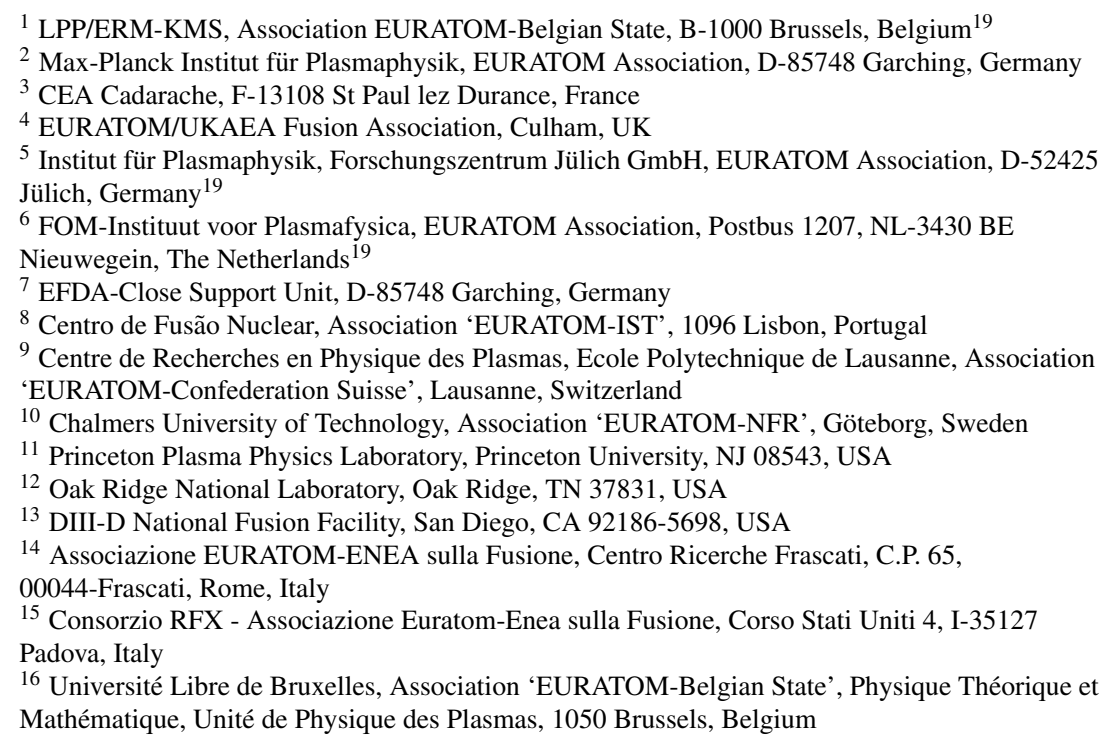

Received 22 June 2001

Published 22 November 2001

Online at stacks.iop.org/PPCF/43/A11

\footnotetext{
${ }^{17}$ Researcher at NFSR Belgium

${ }^{18}$ See Appendix in post-deadline paper at the 18th IAEA Fusion Energy Conference, Sorrento, Italy: J Pamela, 'Overview of recent JET results', IAEA-CN-77-PD/1

${ }^{19}$ Partners in the Trilateral Euregio Cluster (TEC) 


\begin{abstract}
Recent progress towards obtaining high density and high confinement in JET as required for the ITER reference scenario at $Q=10$ is summarized. Plasmas with simultaneous confinement $H_{98(y, 2)}=1$ and densities up to $n / n_{\mathrm{GW}} \sim 1$ are now routinely obtained. This has been possible (i) by using plasmas at high $(\delta \sim 0.5)$ and medium $(\delta \sim 0.3-0.4)$ triangularity with sufficient heating power to maintain Type I ELMs, (ii) with impurity seeded plasmas at high $(\delta \sim 0.5)$ and low $(\delta \leqslant 0.2)$ triangularity, (iii) with an optimized pellet injection sequence, maintaining the energy confinement and raising the density, and (iv) by carefully tuning the gas puff rate leading to plasmas with peaked density profiles and good confinement at long time scales. These high performance discharges exhibit Type I ELMs, with a new and more favourable behaviour observed at high densities, requiring further studies. Techniques for a possible mitigation of these ELMs are discussed, and first promising results are obtained with impurity seeding in discharges at high triangularity. Scaling studies using the new data of this year show a strong dependence of confinement on upper triangularity, density and proximity to the Greenwald limit. Observed MHD instabilities and methods to avoid these in high density and high confinement plasmas are discussed.
\end{abstract}

\title{
1. Introduction
}

The ITER reference operational scenario is the ELMy H-mode with a confinement quality $H_{98(y, 2)}=1$ and beta value $\beta_{\mathrm{N}}=1.8$ at a line-averaged density normalized to the Greenwald density [1] $n / n_{\mathrm{GW}}=0.85\left(\right.$ with $n_{\mathrm{GW}}\left(10^{20} \mathrm{~m}^{-3}\right)=I_{\mathrm{p}}(\mathrm{MA}) /\left(\pi a^{2}\left(\mathrm{~m}^{2}\right)\right)$. Previous experiments in JET and other tokamaks have shown that it becomes increasingly more difficult to keep good confinement properties in the ELMy H-mode at higher densities, usually obtained by gas puff. This year, however, discharges have been obtained at JET with simultaneous high density and high confinement using several techniques: (i) by increasing the triangularity of the plasma to high values $\delta \sim 0.5$, (ii) at medium triangularities $\delta \sim 0.3-0.4$ by increasing the additional heating power so as to keep Type I ELMs, (iii) by impurity seeding in plasmas with both high and low triangularity, (iv) by fuelling the plasma with an optimized pellet injection sequence, and (v) by carefully tuning the gas input, leading to density peaking and densities around or above the Greenwald factor on long time scales. Using these methods or combinations, confinement and density values have been reached as needed for the $Q=10$ ITER reference scenario [2] during quasi-stationary periods of up to $6 \mathrm{~s}$ or up to $15 \times \tau_{\mathrm{E}}$, a duration which was limited by the designed pulse length capabilities of JET. These long-pulse high performance discharges exhibit Type I ELMs, and it is another challenge to mitigate the effect of ELMs, in order to avoid excessive erosion or sputtering of the first wall. Several techniques for ELM mitigation have been studied with and without impurity radiation, and first results indicate possible promising routes to the realization of an operational scenario for ITER that combines high performance with an acceptable heat exhaust. A significant influence of triangularity, density peaking and proximity to the Greenwald density on the scaling of the energy confinement is found. This paper summarizes the main results of experiments undertaken this year on JET, aimed at obtaining simultaneous high confinement and high density in view of ITER. 


\section{High density and high confinement in highly shaped plasmas}

Recent experiments on ASDEX, DIII-D, JET and JT-60 [3-7] have shown the positive influence of triangularity on confinement. This year, the upper and lower triangularity of the plasma, $\delta_{\mathrm{U}}$ and $\delta_{\mathrm{L}}$, have been varied independently in JET to study the influence of these parameters on plasma confinement. A large number of different plasma shapes have been tested. One of them is the so-called 'ITER-like' shape (elongation $\kappa=1.74$ and average triangularity $\left.\delta=\left(\delta_{\mathrm{U}}+\delta_{\mathrm{L}}\right) / 2 \sim 0.45-0.5\right)$. The working gas of the discharges is deuterium with a few per cent $(\sim 2-3 \%)$ of hydrogen. In what follows, the ratio $P_{\text {heat }} / P_{\mathrm{L}-\mathrm{H}}$ of the additional heating power to the $\mathrm{L}-\mathrm{H}$ threshold power will be used as a convenient way to quantify the power level used, as discussed in [8].

ITER-like plasmas (figure 1) simultaneously realize high density up to $\bar{n} / n_{\mathrm{GW}}=1$ with slight peaking $\left(\bar{n} / n_{\text {ped }}=1.2-1.3\right.$, where $\bar{n}$ is the line average over a central chord $(R=3.02 \mathrm{~m})$ and $n_{\text {ped }}$ is the line average over a chord in the edge region $(R=3.75 \mathrm{~m})$ ), high confinement $H_{98(y, 2)} \sim 1$ (with $H_{98(y, 2)}$ the enhancement factor over ELMy H-mode as given by the IPB98 $(y, 2)$ scaling expression, see section 6.1 [2]), and high normalized beta $\beta_{\mathrm{N}}=2$ for quasi-stationary time intervals lasting several seconds (more than $6 \mathrm{~s}$ for the discharge of figure 1). This duration is close to the maximum, technically realizable, flat-top time in JET for this operational scenario. To obtain good confinement conditions in high triangularity scenarios, in addition attention has to be paid to avoid large first ELMs. This was done using gas puffs in the beginning of the discharge. The idea was to maintain the size of the first ELM below the level that creates seed islands for neoclassical tearing modes (NTM), as mode numbers $m / n=3 / 2$ are often observed after large ELMs.
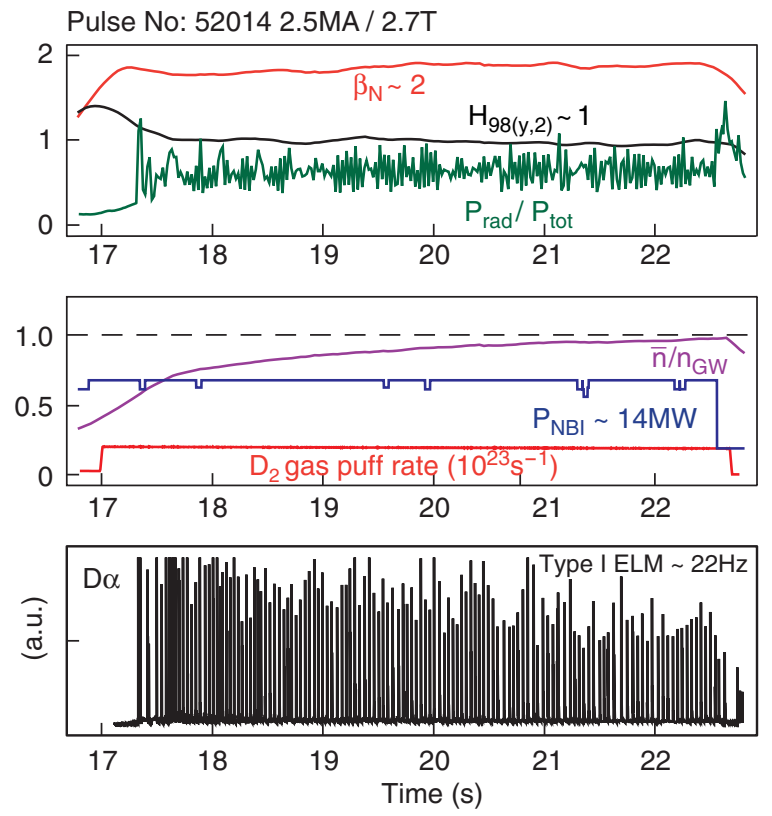

Figure 1. Time traces of an ITER-like JET plasma (\#52014, 2.5 MA/2.7 T) obtained at high triangularity $(\delta \sim 0.47)$ showing simultaneously realization $\bar{n} / n_{\mathrm{GW}}>0.85, \beta_{\mathrm{N}} \sim 2$ and $H_{98(y, 2)} \sim 1$ from about $t=20$ s onwards. 


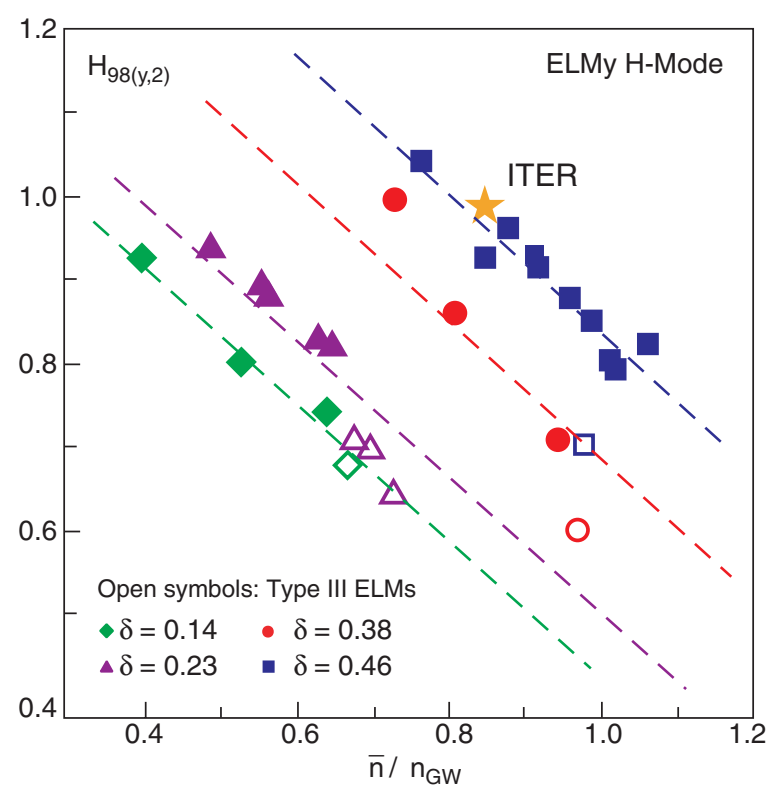

Figure 2. Plot of the enhancement factor $H_{98(y, 2)}$ versus the Greenwald factor $\bar{n} / n_{\mathrm{GW}}$ in discharge series with different triangularities and no impurities. Data for $\delta$ up to 0.38 have been obtained in the MkII JET divertor [9]. The highest triangularity data $(\delta=0.46)$ have been obtained this year in the gasbox divertor (MkIIGB) configuration. The projected ITER design point is indicated by the star. Open symbols correspond to discharges with Type III ELMs.

Typically, such plasmas have low frequency Type I ELMs [9]. At all triangularities, there is a confinement degradation with density but higher triangularities achieve higher densities with good confinement (figure 2). Data from ITER-like plasmas now encompass the ITER design point $\left(\bar{n} / n_{\mathrm{GW}}=0.85\right.$ and $\left.H_{98(y, 2)}=1\right)$.

High confinement was also obtained with strong gas puff in lower triangularity plasmas ( $\delta \sim 0.3$ ) by increasing the heating power sufficiently to maintain Type I ELMs. This was done for two sets of discharges (figure 3$)$ at medium triangularity $(\delta=0.33)$ at low and high values for $P_{\text {heat }} / P_{\mathrm{L}-\mathrm{H}}\left(1.6<P_{\text {heat }} / P_{\mathrm{L}-\mathrm{H}}<2.1\right.$ and $\left.2.4<P_{\text {heat }} / P_{\mathrm{L}-\mathrm{H}}<4.2\right)$. The higher values for $P_{\text {heat }} / P_{\mathrm{L}-\mathrm{H}}$ maintained higher confinement even with strong gas puff (leading to high densities up to the Greenwald limit), while lower $P_{\text {heat }} / P_{\mathrm{L}-\mathrm{H}}$ led to degradation of confinement with high gas puff rates, as illustrated in figure 3 . The confinement degradation is correlated with a change from Type I to Type III ELMs and only densities below the Greenwald limit are reached.

Although the high density in these plasmas was reached by high gas fuelling rates, $H_{98(y, 2)}$ degraded by only 5-10\% compared to the unfuelled case. The decreased sensitivity of energy confinement to high gas puff rates of this type of discharges was explained in [9] in part by stabilization of ballooning mode turbulences in the edge due to triangularity. The net result are plasmas with high stored energy and normalized beta value, high plasma density around the Greenwald limit and consequently low plasma dilution characterized by a $Z_{\text {eff }} \sim 1.5$.

\section{High density and high confinement impurity seeded plasmas}

Experiments with impurity seeding on TEXTOR, DIII-D, JET and JT-60U [10] have shown that it is possible to combine or even improve the confinement and density properties of 


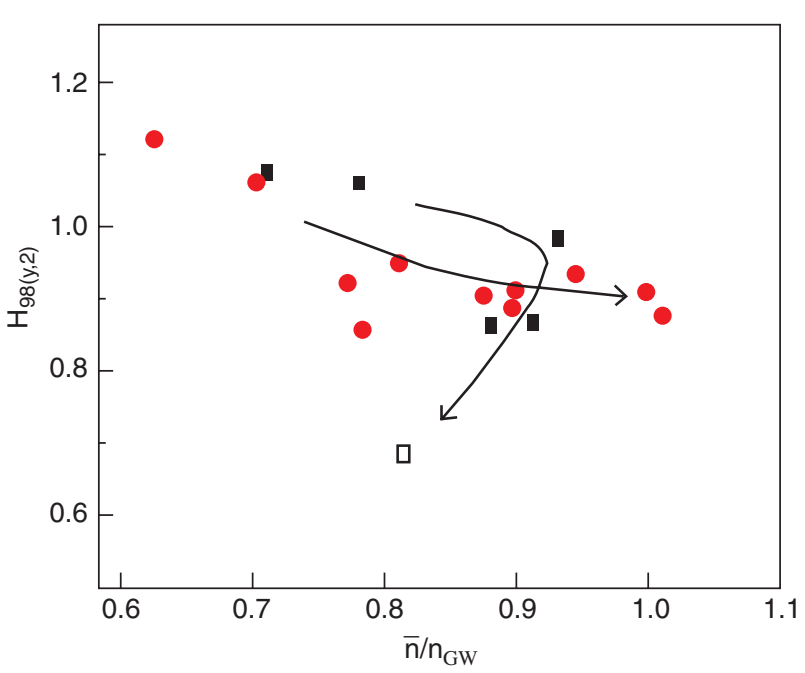

Figure 3. The enhancement factor $H_{98}(y, 2)$ for medium triangularity plasmas $(\delta \sim 0.3)$ as a function of the Greenwald number. Circles correspond to discharges at $1.9 \mathrm{MA} / 2.0 \mathrm{~T}$ with $P_{\text {heat }} / P_{\mathrm{L}-\mathrm{H}}>$ 2.4 , squares to discharges at $2.4 \mathrm{MA} / 2.4 \mathrm{~T}$ and $1.9 \mathrm{MA} / 2.0 \mathrm{~T}$ with $1.6<P_{\text {heat }} / P_{\mathrm{L}-\mathrm{H}}<2.4$. Open symbol indicates Type III, solid symbols Type I ELMs. Arrows show the direction of increasing gas puff rates. For the second class of discharges, confinement and density is reduced at high gas puff rates, accompanied by the appearance of Type III ELMs.

plasmas (leading to high confinement, $H_{98(y, 2)} \sim 1$, and high density, $\bar{n} / n_{\mathrm{GW}} \sim 1$ ) with high levels of radiation in the edge. Experiments with radiative mantle plasmas started in JET in 1999 [11] have been continued this year [12-18] in (i) low triangularity discharges with the $\mathrm{X}$-point lowered onto or just inside the material barrier (called septum or dome) dividing the inboard and outboard sides of the divertor, resulting in the so-called 'septum' configuration, and (ii) discharges at high triangularity with a geometry close to the ITER one.

\subsection{Impurity seeding in 'septum' discharges}

This discharge type is mid-way between a divertor and a pumped limiter configuration. Surprisingly, these discharges have a lower H-mode threshold power than discharges without septum [19] and thus remain in H-mode even at high radiation levels. Impurity seeding has been applied to both low $(\delta=0.2, \kappa=1.6)$ and medium triangularity $(\delta=0.3, \kappa=1.72)$ discharges in the septum configuration (figure 4 ). About $0.5 \mathrm{~s}$ after the application of additional heating, a strong gas puff was applied. Confinement was reduced during the gas puff phase, as seen in the reduction of the enhancement factor $H_{98(y, 2)}$ to about 0.8. During the $\mathrm{D}_{2}$ puff phase, Ar was also puffed and both the radiative power level and plasma density increased. After the $\mathrm{D}_{2}$ gas fuelling valve closed, the density was constant $\left(\bar{n} / n_{\mathrm{GW}} \sim 0.9\right)$ and the confinement was restored to pre-puff values $\left(H_{98(y, 2)} \sim 1\right)$, with radiation fractions $P_{\text {rad }} / P_{\text {tot }}$ around $50 \%$. In this way, values are obtained for the product $H_{98(y, 2)} \times \bar{n} / n_{\mathrm{GW}} \sim 0.9$ at $\bar{n} / n_{\mathrm{GW}} \sim 0.9$, outstanding for low triangularity plasmas. Those values can be maintained stationary for $5 \mathrm{~s}$ or about $12 \tau_{\mathrm{E}}$. This phase (from $20 \mathrm{~s}$ onwards in figure 4 ) is called the 'afterpuff' phase. During the afterpuff phase, gentle $\mathrm{Ar}$ and $\mathrm{D}_{2}$ refuelling was applied keeping the radiation level and the plasma density constant. Only $15-20 \%$ of the input power was radiated in the divertor, with about $30 \%$ of the input power radiated in the edge of the main plasma chamber. Ar seeding is 


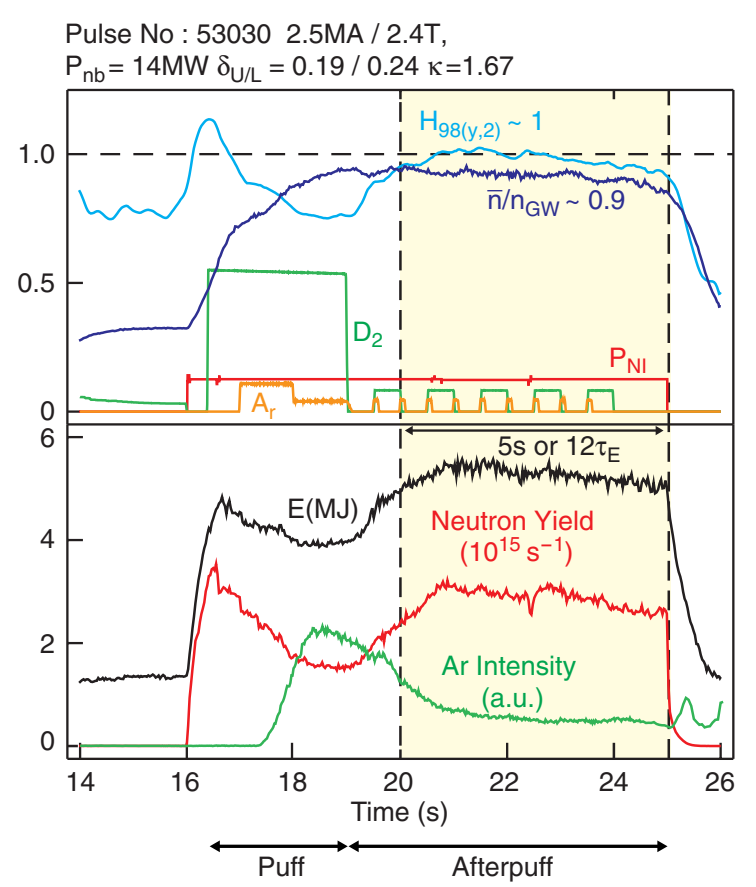

Figure 4. Ar seeding in a low triangularity $(\delta \sim 0.22)$ ELMy H-mode plasma with the X-point on the septum. Time evolution of the enhancement factor $H_{98}(y, 2)$, the Greenwald factor, neutron yield, plasma stored energy, bulk radiation and deuterium and Ar injection rate. The main puff phase consists of D and Ar injection, intended to increase both the density and radiation level in the discharge. The 'afterpuff' phase (with reduced levels for $\mathrm{D}_{2}$ and Ar puff) had simultaneous high confinement and high density.

more adapted to the JET plasma parameters than Ne, as the latter leads to a higher $Z_{\text {eff }}$ for a given radiation level.

Note that the electron density increase not only results from the extra electrons provided by the ionization of the seeded Ar but genuine changes must take place in the particle confinement time $\tau_{\mathrm{p}}$, the fuelling efficiency (as defined in [20]) or both in the presence of Ar.

The same technique (puff and afterpuff) was applied in septum discharges at medium triangularity $(\delta \sim 0.3$ ), leading to similar results. At $\delta \sim 0.3$ higher densities were obtained with D puff only, whereas at $\delta=0.2$ similar densities could only be obtained with additional Ar seeding during the $\mathrm{D}$ puff phase. Both low and high triangularity discharges with impurity seeding showed a peaking of the electron density profile.

The ELM frequency reduced during Ar seeding for a given (high) density and edge pedestal. Consequently smaller time integrated ELM losses occurred. This will be further discussed in section 7. Nevertheless, fundamental changes in bulk confinement must also take place, as the Ar seeding also correlated with $\chi_{\text {eff }}$ (calculated by TRANSP [21]) dropping in the core [10]. Such an improvement of confinement has been demonstrated in L-mode divertor plasmas with impurity seeding in DIII-D and JET [38].

\subsection{Impurity seeded ITER-like plasmas}

Ar seeded ITER-like plasmas (figures 5(a) and $(b)$ ) showed a radiating mantle and $P_{\text {rad }} / P_{\text {tot }}=$ 60-70\%. ICRH has been applied to avoid impurity accumulation (see section 3.3). Ar seeding 


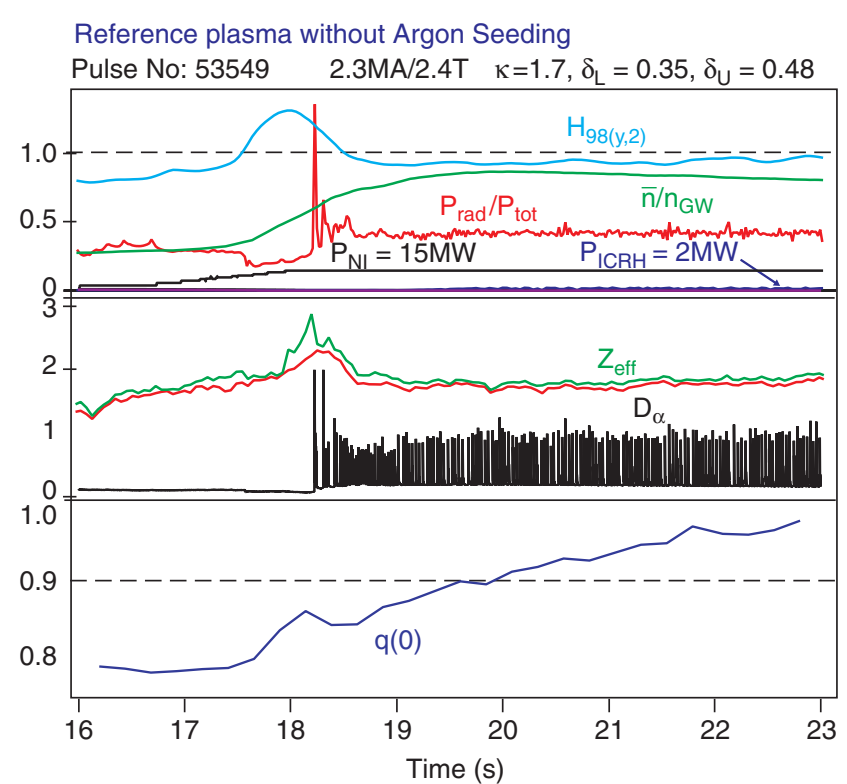

Argon Seeded plasma

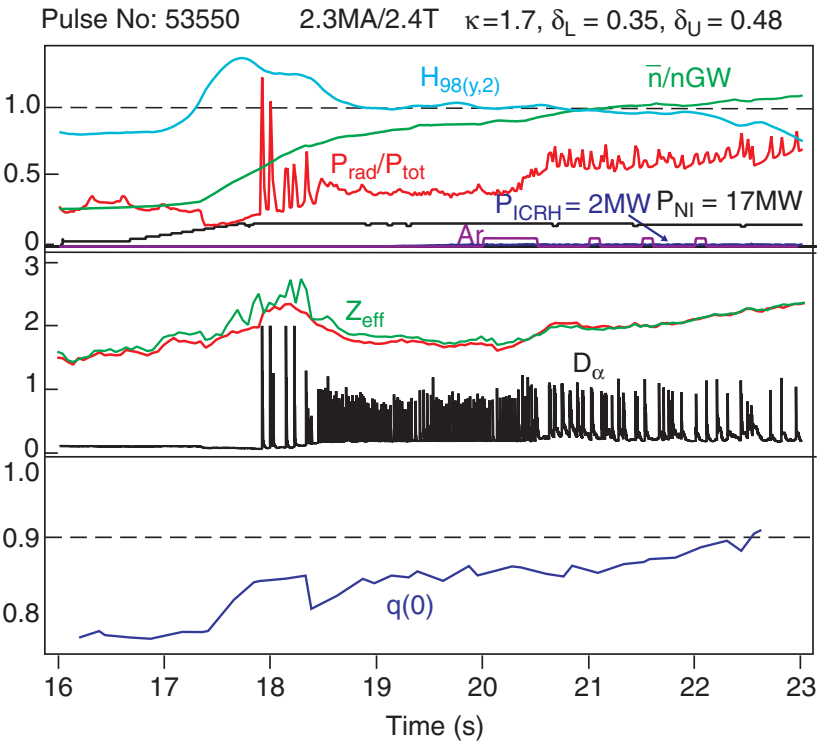

Figure 5(a). Ar seeding in a high triangularity ( $\delta \sim 0.47)$ ELMy H-mode plasma. Temporal evolution of confinement parameters, central $q$ value, $\mathrm{D}_{\alpha}$ intensity and $Z_{\mathrm{eff}}$. The non-seeded reference plasma (top, \#53549) and plasma with Ar seeding (bottom, \#53550) were both NBI heated (15 MW, respectively $17 \mathrm{MW}$ ) with additional $2 \mathrm{MW}$ of centrally deposited ICRH (H-minority heating). Ar seeding in $\# 53550$ started at $t=20 \mathrm{~s}$, and radiation fractions $P_{\mathrm{rad}} / P_{\text {tot }} \sim 70 \%$ were achieved.

correlates with an increase in the plasma density, up to $1.2 \times n_{\mathrm{GW}}$ (figure $5(a)$ ). Confinement was unchanged in the Ar seeded discharge $\left(H_{98(y, 2)} \sim 1\right)$. $Z_{\text {eff }}$ is somewhat increased from $\sim 1.7$ to $\sim 2.0$. Further optimization of these discharges is foreseen with respect to the deuterium and Ar refuelling, in order to further increase the stationarity and the product $H_{98(y, 2)} \times \bar{n} / n_{\mathrm{GW}}$. 

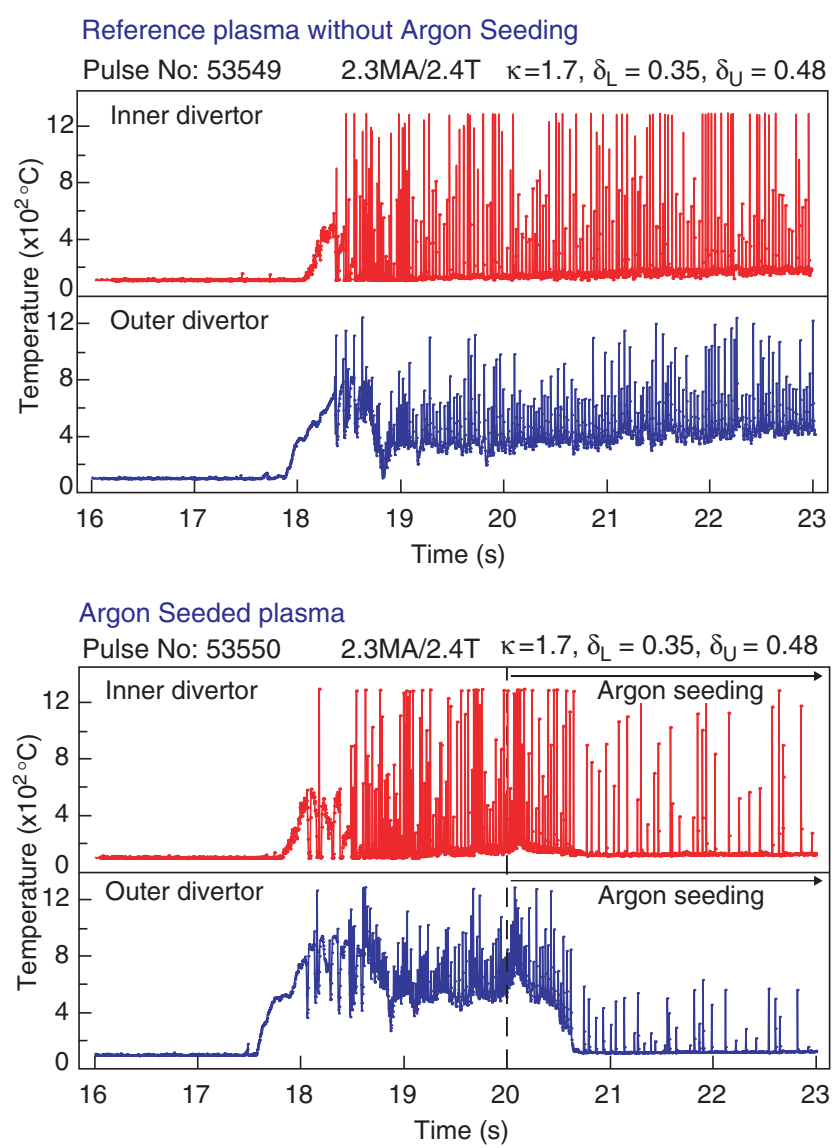

Figure 5(b). Infrared measurements of the JET divertor target plates for the same discharges as figure 5(a).

Infrared measurements at a fixed location in the inner and outer divertor (figure 5(b)) showed a reduction in the surface temperature of the divertor target with Ar seeding. The effect is clearly visible in the inner divertor, and even more in the outer divertor, where the base level drops from about $500^{\circ} \mathrm{C}$ to about $100^{\circ} \mathrm{C}$ and also the maximum temperature spikes due to ELMs are reduced from $1300^{\circ} \mathrm{C}$ to $600^{\circ} \mathrm{C}$ [22]. Although these results are from first IR thermographic measurements in such a scenario and further work is needed to consolidate these findings, the result looks promising as a possible technique for ELM mitigation.

\subsection{Application of central heating in impurity seeded plasmas to optimize the impurity content}

Sawteeth play an important role in maintaining a low dilution in the plasma centre of impurity seeded plasmas. In ELMy H-modes without impurity seeding, generally a slow increase in the central safety factor $q(0)$ is seen (see figure 5(a)). With excessive Ar injection rates, the following four effects are observed: (i) further increase in the central $q$ leading to sawteeth disappearance when $q(0)>1$ followed by impurity accumulation, (ii) an increase in MHD activity [15] which can lead to (iii) a decrease in the energy confinement time and 


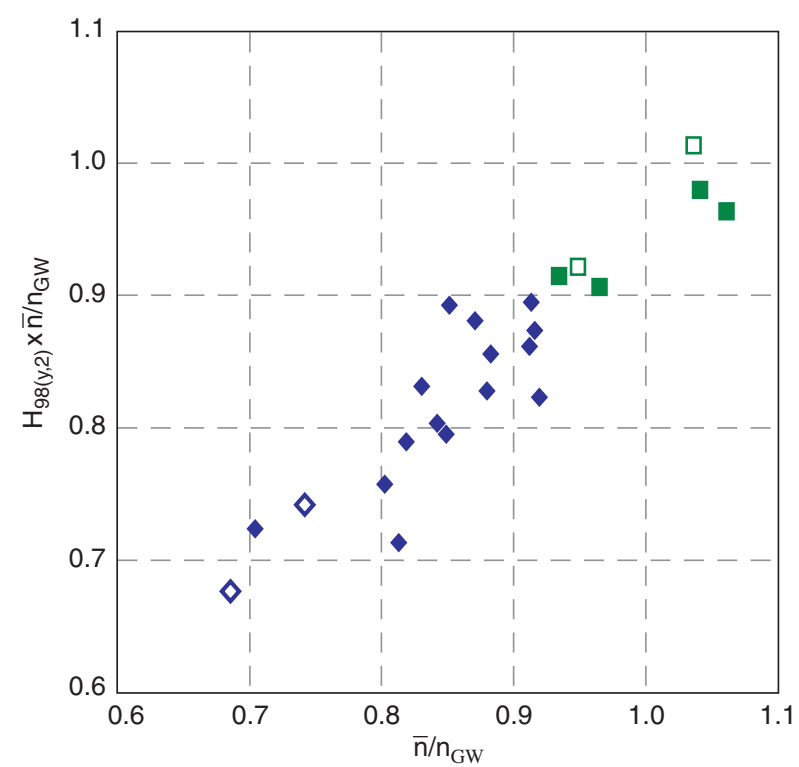

Figure 6. Confinement plotted versus Greenwald number for Ar seeded plasmas (solid symbols) and reference cases (open symbols). Values are obtained by averaging over time intervals of $1.5 \mathrm{~s}$ (about $3 \tau_{\mathrm{E}}$ for the discharges considered). Data belong to two distinct discharge types: (i) with the $\mathrm{X}$-point on the septum at low $\delta=0.15$ (diamonds), and (ii) ITER-like discharges at high $\delta=0.45$ (squares).

(iv) appearance of ELM free phases. The causality between these effects needs further study, but the current understanding is that the changes in the central part of the $q$ profile induce the others. Central heating is one means to counteract the modifications in the $q$ profile and to keep $q(0)<1$, in order to keep the sawtooth MHD. At the higher densities reached in these discharges, the energy deposition of the beams becomes flatter and even hollow. Therefore, in addition to NBI heating, ICRH (H-minority heating) is applied with the resonance layer located on axis, at moderate heating levels (1-2 MW) to avoid sawtooth stabilization by RF induced fast ions. The central electron temperature increased, and the central $q$ remained below unity (figure 5(a)). As a result, the sawteeth remained and core impurity accumulation did not occur. Note that sawtooth activity reduces the central impurity content without increasing the impurity content in the rest of the plasma, as deduced from $\mathrm{Ar}$ concentration and $Z_{\text {eff }}$ profiles.

An $H_{98(y, 2)} \times \bar{n} / n_{\mathrm{GW}}$ versus $\bar{n} / n_{\mathrm{GW}}$ diagram (figure 6 ) shows that low triangularity plasmas with Ar seeding had $20 \%$ higher $n \tau_{\mathrm{E}}$ than unseeded plasmas, while ITER shaped plasmas with Ar had similar $n \tau_{\mathrm{E}}$ as unseeded plasmas. Thus, Ar seeding generally allows one to combine good confinement properties with high radiation levels in a mantle around the plasma. Further study is needed to reveal the physical mechanisms behind the confinement improvement with $\mathrm{Ar}$ in the low triangularity discharges.

\section{Slow density profile evolution}

For a given line-averaged density and temperature profile, fusion power increases with increased peaking of the plasma density, thus optimizing the fusion yield in a reactor. Several tokamaks have observed density peaking on long time scales [23,24]. JET [25] has also recently observed similar density peaking on long time scales for various plasma currents 

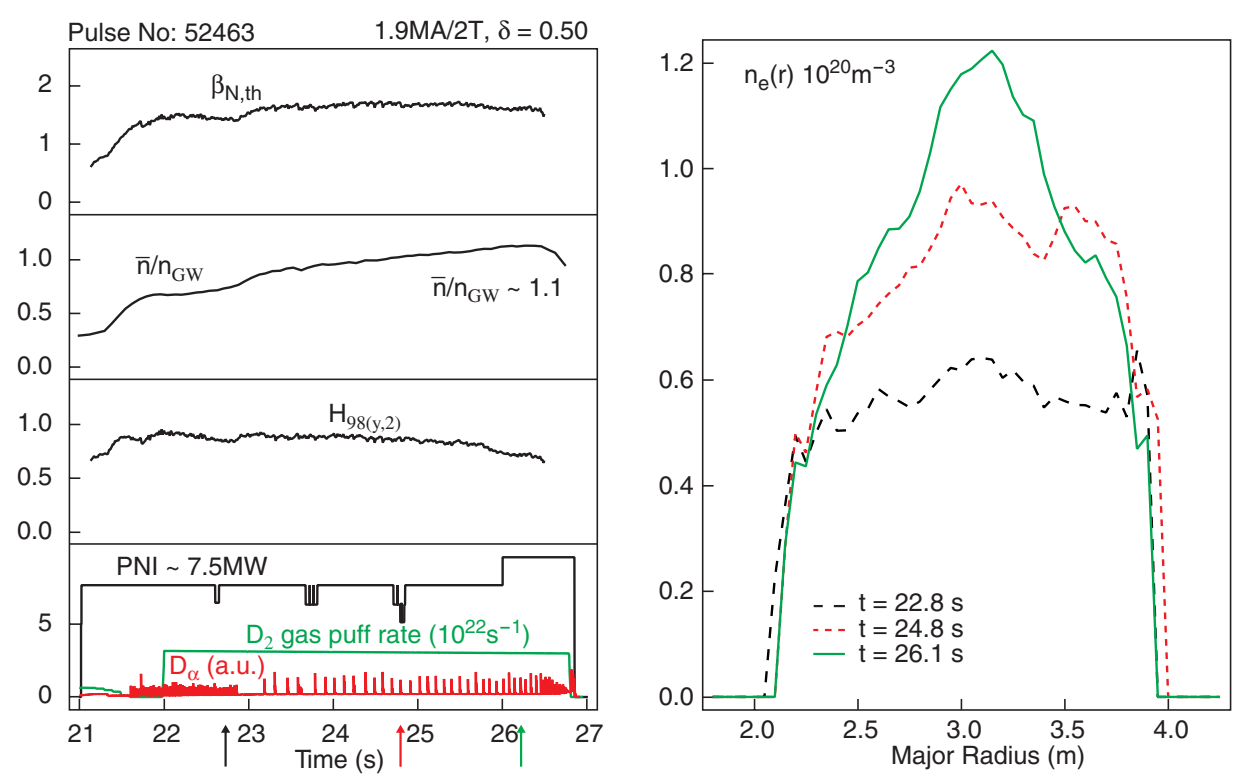

Figure 7(a). Time evolution and density profiles of a high triangularity $(\delta=0.5)$ discharge with spontaneous density peaking, heated with $7.5 \mathrm{MW}$ of NBI. Careful gas puff is applied, leading to values of the density up to $\bar{n} / n_{\mathrm{GW}} \sim 1$, at nearly constant values for $\beta_{\mathrm{N}} \sim 1.7$, but with a slightly degrading $H_{98(y, 2)}$ with increasing density. Density profiles are shown for the times indicated by the arrows.

$\left(0.95 \leqslant I_{\mathrm{p}} \leqslant 2.5 \mathrm{MA}\right)$ and plasma shapes. Outboard and inboard divertor fuelling as well as main chamber fuelling have been tried, with highest densities obtained when fuelling the discharge in the private region from the inboard base ring in the divertor.

A key ingredient of these experiments is the use of gas fuelling rates below the values corresponding to the degradation of the edge transport barrier. Figure 7( $a$ ) shows a plasma where the line-averaged density monotonically increased over a period of several seconds, reaching 1.1 times the Greenwald density after $5 \mathrm{~s}$, followed by a roll-over in confinement. Plasma density profiles illustrate the evolution from a nearly flat profile to a strongly peaked density profile at the end of the discharge, with $\bar{n} / n_{\text {ped }} \sim 1.6$.

At these lower heating powers $\left(P_{\text {heat }}<8 \mathrm{MW}\right)$, the current profile broadens, leading to $q(0)$ values above 1 and the disappearance of sawtooth MHD followed by collapse of the central temperature profile due to the accumulation of (metallic) impurities in the centre accompanied by increased core radiation. If centrally deposited ICRH is applied to avoid the loss of sawteeth, one obtains discharges with high values of the Greenwald factor (up to $\left.\bar{n} / n_{\mathrm{GW}}=1.1\right)$ but with density profiles showing a moderate peaking $\left(\bar{n} / n_{\text {ped }}=1.2-1.3\right)$ and reduced confinement $\left(H_{98(y, 2)} \sim 0.6\right)$. Higher NBI heating powers also keep sawtooth MHD but with less confinement degradation. Avoidance of MHD modes was attempted using optimized gas puff in the beginning of the discharge, usual for high $\delta$ plasmas, as explained in section 1 . In this way, discharges could be realized with a quasi-stationary phase of over $3 \mathrm{~s}$ (terminated by the end of the heating pulse) at $\bar{n} / n_{\mathrm{GW}}=1, H_{98(y, 2)}=0.96$, $\beta_{\mathrm{N}}=2$ with sawteeth preserved and stationary peaked density profiles with $\bar{n} / n_{\text {ped }} \sim 1.3$ (figure 7(b)).

The physical mechanisms leading to the density peaking are not yet fully understood and require further study. 

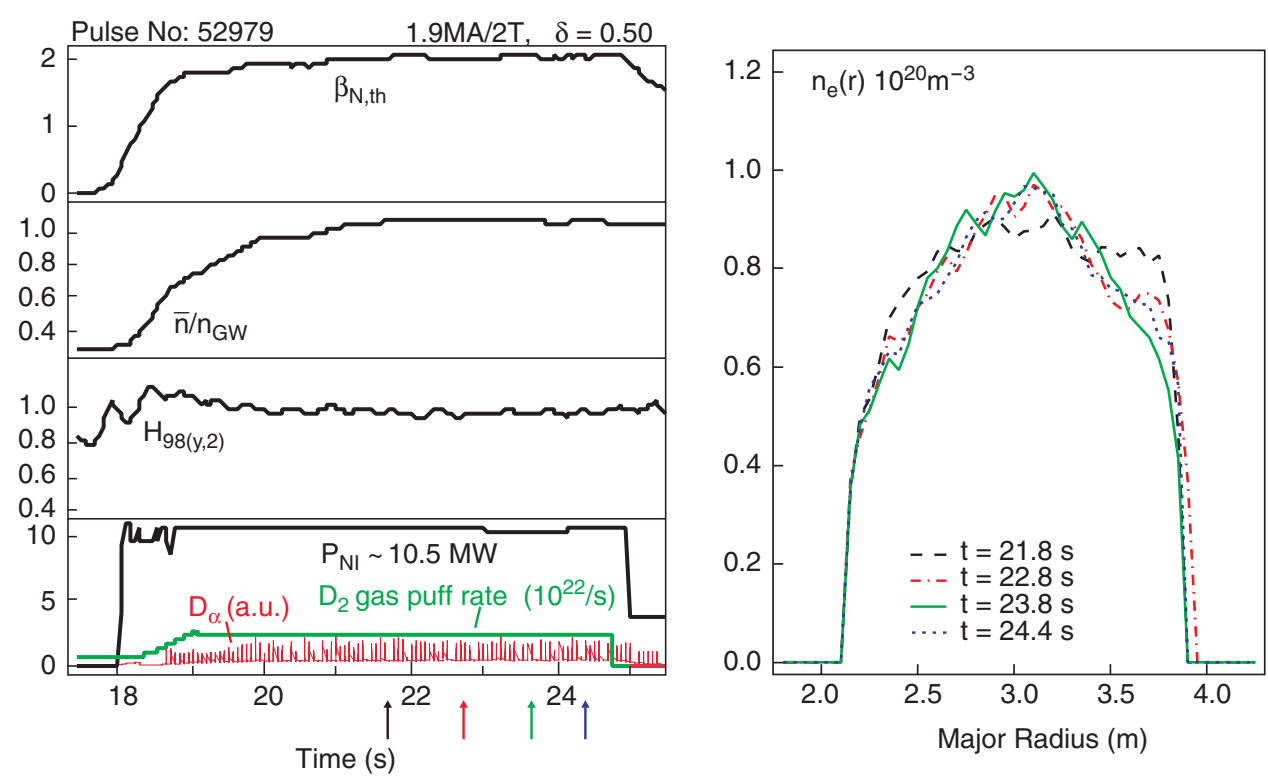

Figure 7(b). Time evolution and density profiles of a high triangularity $(\delta=0.5)$ discharge with spontaneous density peaking, heated with $10.5 \mathrm{MW}$ of NBI. Gas puff is applied, leading to values of the density up to $\bar{n} / n_{\mathrm{GW}} \sim 1$, at nearly constant values for $\beta_{\mathrm{N}} \sim 2$ and $H_{98(y, 2)} \sim 1$. Density profiles are shown for the times indicated by the arrows.

\section{Pellet injection}

Pellet fuelling experiments have been conducted [26] to achieve long pulse operation at densities in the vicinity of the Greenwald density with good energy confinement. A target plasma configuration with $\delta \sim 0.34$ and at $2.5 \mathrm{MA} / 2.4 \mathrm{~T}$ was chosen, showing confinement collapse when trying to fuel the density beyond $\bar{n} / n_{\mathrm{GW}}=0.8$ with gas puff only. Solid $4 \mathrm{~mm}^{3}$ deuterium cubes were launched at a speed of $160 \mathrm{~m} \mathrm{~s}^{-1}$ into the plasma from the high field side via an injection tube tilted by $44^{\circ}$ with respect to the horizontal plane and with a tangency radius at a normalized minor radius $\rho \sim 0.6-0.7$. An optimized pellet fuelling scenario should minimize or avoid the following effects: (i) prompt particle losses causing too strong an increase of the neutral gas pressure and edge density, (ii) trigger of MHD activity by the pellet, and (iii) ELM bursts following pellet injection. Each of these pellet related effects can cause severe energy losses from the plasma.

Mitigation of the confinement losses resulting from pellet induced ELMs was achieved with an adapted pellet fuelling cycle consisting of interrupting the injected pellet string. This leads to recovery of the plasma energy content, while the particle inventory still remains high. The finally optimized pellet sequence is composed of an initial density build-up phase at a pellet injection rate of about $6 \mathrm{~Hz}$ followed by a phase where the density is sustained at an injection rate of about $2 \mathrm{~Hz}$. In this way, a density increase beyond the Greenwald limit was achieved without significant persistent loss of the plasma energy content, as illustrated in figure 8 . The initial pellet sequence causes the expected energy drop due to enhanced ELM activity. The pellet string is then interrupted for about $0.5 \mathrm{~s}$ to allow for energy recovery, followed by a string at $2 \mathrm{~Hz}$. With this reduced repetition rate, the transient energy drop initiated by every pellet can be almost fully recovered before the next pellet arrives in the plasma. Moreover, successive pellets gradually increase the plasma density until finally a 


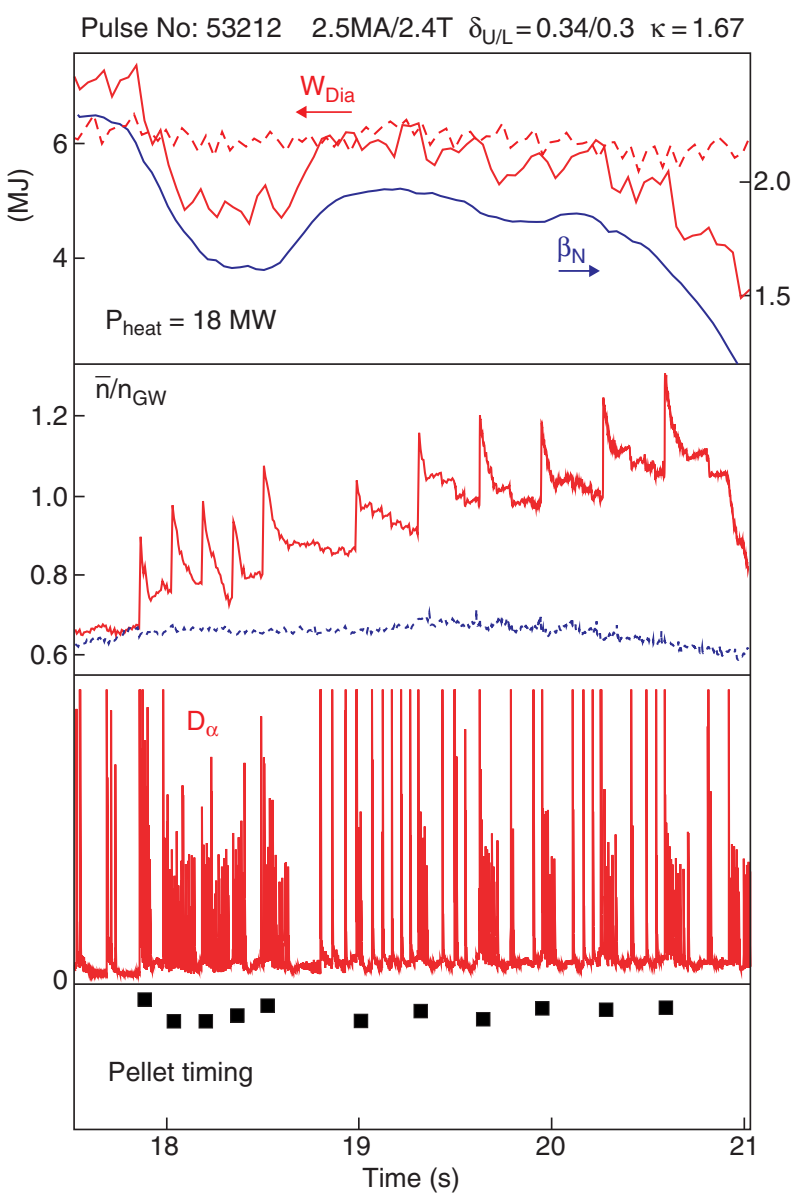

Figure 8. Time evolution of the diamagnetic energy, normalized beta, Greenwald factor and the intensity of the $\mathrm{D}_{\alpha}$ line for a medium triangularity JET discharge with an optimized pellet fuelling cycle. Squares indicate the timing sequence of the pellets. Solid lines correspond to the discharge with pellets, dashed lines to a reference discharge without pellets (and without gas puff).

density above the Greenwald limit is achieved with a simultaneous high plasma energy content of about $6 \mathrm{MJ}$ and $\beta_{\mathrm{N}}>1.8$, corresponding to an $H_{98(y, 2)} \sim 0.82$. The energy and central lineaveraged density of a reference discharge without gas puff or pellet injection are indicated by the dashed lines in the figure for comparison. It is clear that the discharge with the optimized pellet injection obtains the same energy content, however, at a much higher density as the non-fuelled reference. Further experiments are planned next year aimed at improving the stationarity of these discharges.

A too high pellet injection rate can lead to severe cooling of the central plasma temperature leading to reduction of the critical beta (because of the decrease of the ion gyroradius due to the reduction in temperature) and therefore renders the plasma sensitive to the appearance of NTMs. To keep the plasma temperature sufficiently high, dominant NBI heated plasmas were also heated with centrally deposited ICRH, resulting indeed in reduction of the MHD activity. Raising the toroidal field is also beneficial to reduce MHD, but leads for a fixed power on the contrary to Type III ELMs and reduced plasma performance. 


\section{Confinement scaling of high triangularity discharges and comparison of recently obtained confinement data to the ITER design point parameters}

\subsection{Influence of triangularity, density peaking and the Greenwald factor on the enhancement factor $H_{98}(y, 2)$}

The new results described in the previous sections permit the investigation of the effect of triangularity and density peaking on confinement. The data obtained are compared to the standard IPB98 $(y, 2)[2]$ scaling law:

$$
\tau_{\mathrm{E}, \mathrm{IPB} 98(y, 2)}=0.0562 I_{\mathrm{p}}^{0.93} B_{\mathrm{t}}^{0.15} \bar{n}^{0.41} P^{-0.69} A_{\mathrm{i}}^{0.19} R^{1.97} \varepsilon^{0.58} \kappa^{0.78}
$$

with the usual units (s, MA, T, $10^{20} \mathrm{~m}^{-3}$, MW and $\mathrm{m}$ ). The residuals of the factor $H_{98(y, 2)}=$ $\tau_{\mathrm{E}, \exp } / \tau_{\mathrm{E}, \mathrm{IPB} 98(y, 2)}$ for a subset of the data which had accurate values of the pedestal density $n_{\text {ped }}$ (obtained from the interferometer channel at $R=3.75 \mathrm{~m}$ in JET) have been fitted to the upper triangularity, the Greenwald factor and the density peaking factor $\bar{n} / n_{\text {ped }}$. This resulted

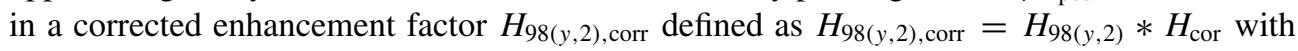
$H_{\text {cor }}=0.84+0.17 \delta_{\mathrm{U}}-0.13 \bar{n} / n_{\mathrm{GW}}+0.50\left(\bar{n} / n_{\text {ped }}-1\right)$. This expression shows clearly the increase of confinement with triangularity $\delta_{\mathrm{U}}$ and density peaking $\bar{n} / n_{\text {ped }}$ and the degradation with increasing $\bar{n} / n_{\mathrm{GW}}$. More refined expressions can be used [27], leading essentially to the same conclusion. For an illustration of the influence of density peaking on the extrapolation to ITER, we take the ITER design point $\left(\delta_{\mathrm{U}}=0.5, \bar{n} / n_{\mathrm{GW}}=0.85, H_{98(y, 2)}=1\right)$. Without peaking, a confinement factor $H_{98(y, 2) \text {, corr }} \sim 0.81$ is found. With a modest density peaking $\left(\bar{n} / n_{\text {ped }}=1.3\right)$, this rises to $H_{98(y, 2), \text { corr }} \sim 0.96$.

\subsection{Comparison of confinement data to the ITER design point}

The present data, reaching ITER values for $H_{98(y, 2)}$ and $\bar{n} / n_{\mathrm{GW}}$, have a 2-3 times larger normalized gyroradius $\rho^{*}$, and 10 times larger collisionality $v^{*}$ (figures $9(a)$ and $(b)$ ).

Although it is not possible with JET or any existing device to reach simultaneously $\beta, v^{*}$ and $\rho^{*}$ as in ITER, only JET can generate $\beta, v^{*}$ and $\rho^{*}$ data which are closest to dimensionless ITER parameters. First, one can match simultaneously ITER $v^{*}$ and $\beta_{\mathrm{N}}$ values in JET, by operating at lower density with $\bar{n} / n_{\mathrm{GW}} \sim 0.3-0.5$, i.e. the normal operating density range in the absence of gas puff or pellet injection. Second, as $\rho^{*} \propto B^{-2 / 3} a^{-5 / 6}$ [2] for fixed $\beta$ and $\nu^{*}$, operation at high magnetic fields (together with the large machine dimensions of JET) gives the smallest $\rho^{*}$ values possible at this moment in any device. Experiments on JET are needed at higher field and current to operate closer to the $\rho_{\text {ITER }}^{*}$ and hence improve the preparation for the ITER operational scenario. To carry out such a programme, that is operating in excess of 4.0 MA at the ITER $\beta$ and $v^{*}$, means that a total heating power in excess of $30 \mathrm{MW}$ will be required. It is planned that this level of power will be available towards the end of 2002 .

\section{ELMs in high density, high confinement plasmas and possible mitigation techniques}

High density, high confinement plasmas need a heat exhaust mechanism compatible with the first wall to be useful for a future fusion reactor. The power loss per ELM for ITER operating at $Q=10$ and $400 \mathrm{MW}$ fusion power cannot be larger than $\sim 1 \%$ of the plasma stored energy, in order to avoid melting of tungsten and evaporation of carbon target materials [28]. Two effects are important when discussing the power losses by ELMS: (i) the peak power flux during the ELM itself, and (ii) the power flux averaged over many ELMs. High peak power loss during ELMs can cause serious damage because of ablation and/or melting of the target 


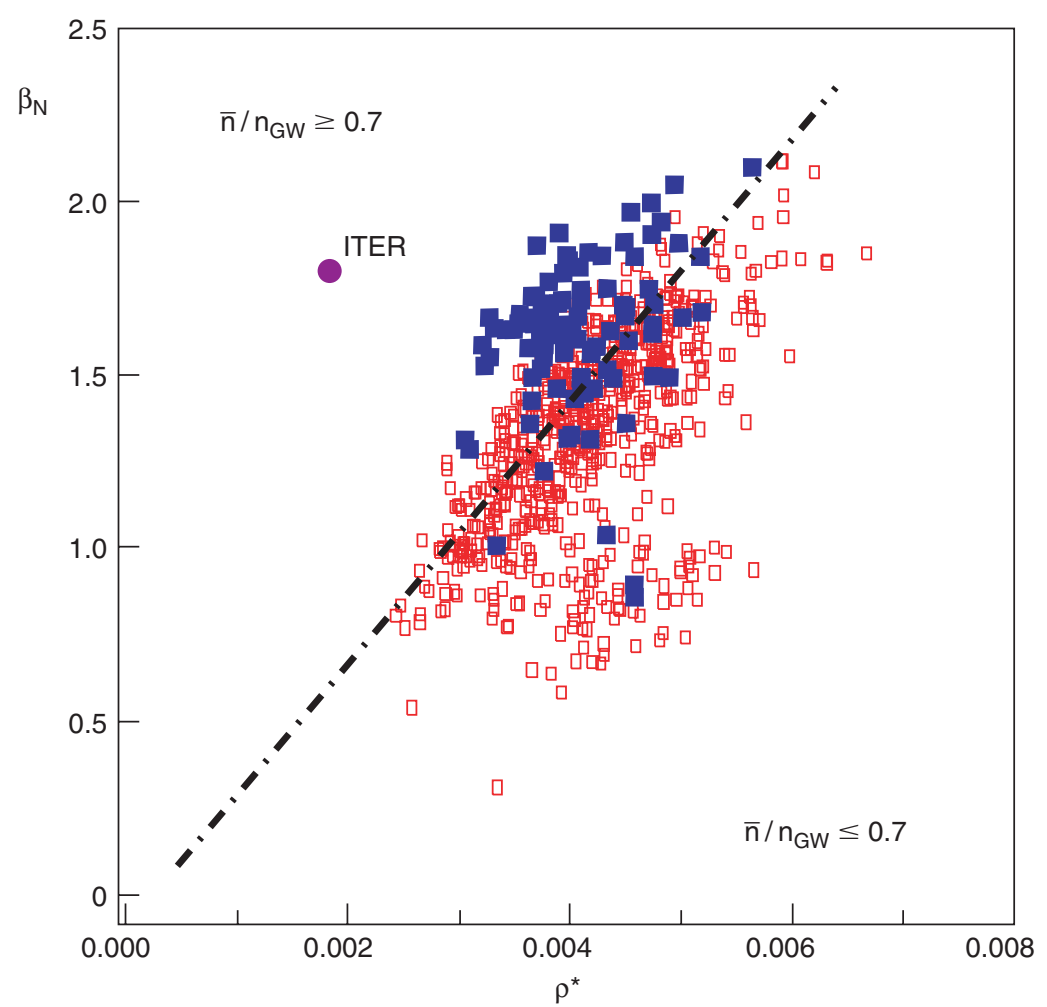

Figure $9(\boldsymbol{a})$. Normalized gyroradius $\rho^{*}$ plotted versus normalized beta $\beta_{\mathrm{N}}$ for JET ELMy H-mode discharges since 1994 with $1.8 \mathrm{MA}<I_{\mathrm{p}}<2.7 \mathrm{MA}$. Data are from the JET steady-state database. Open symbols correspond to data obtained before 2000, solid symbols to those from 2000-2001. The dashed-dotted line roughly divides this diagram in a region below and above $\bar{n} / n_{\mathrm{GW}}=0.7$. The ITER design point is indicated by the solid circle.

material. However, for given energy loss during individual ELMs, a reduction of the ELM frequency can increase the divertor target lifetime.

Several approaches are under investigation on different machines to find a solution for the power load on the first wall during ELMs: (i) the realization of so-called Type II ELMs [29,30], (ii) regimes with enhanced $\mathrm{D}_{\alpha}$ radiation [31], and (iii) impurity seeding [10].

JET discharges at high and medium triangularity with heating powers sufficient to keep Type I ELMs show interesting properties. With increasing gas puff and a corresponding increase in the edge density up to $n_{\text {ped }}=75 \% n_{\mathrm{GW}}$, the frequency of the ELMs first shows a mild increase and then drops again. At extreme gas puff rates, Type III ELMs appear. Measurements show that above a pedestal density $n_{\text {ped }} \sim 75 \% n_{\mathrm{GW}}$ the pedestal electron temperature no longer decreases with increasing pedestal electron density, but stays more or less constant and above the critical temperature for transition to Type III ELMs. In other words, the decrease of the pedestal pressure with density $\left(\nabla p_{\text {ped }} \propto 1 / n^{2}\right)$, usually seen at lower triangularity, is no longer observed in these high and medium triangularity high confinement plasmas. The net effect of the higher triangularity is thus a larger operational space for the existence of Type I ELMs at high density. First modelling attempts [32] for the edge stability with the JETTO code coupled to the infinite- $n$ ideal ballooning stability code IDBALL [33] show second stability access for ideal ballooning for part of the edge pedestal of the plasmas 


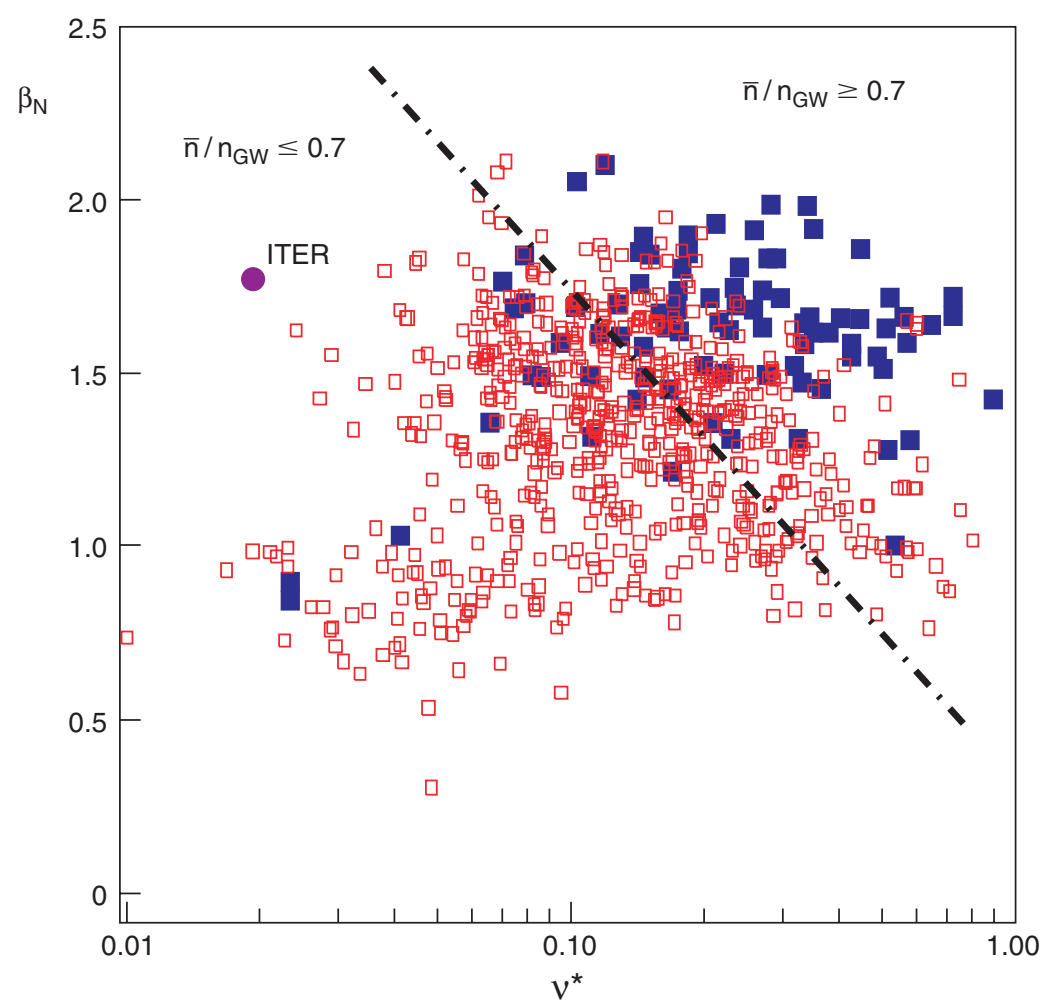

Figure 9(b). Normalized gyroradius $\rho^{*}$ plotted versus collisionality $v^{*}$ for JET ELMy H-mode discharges since 1994 with $1.8 \mathrm{MA}<I_{\mathrm{p}}<2.7 \mathrm{MA}$. Data are from the JET steady-state database. Open symbols correspond to data obtained before 2000, solid symbols to those from 2000-2001. The dashed-dotted line roughly divides this diagram in a region below and above $\bar{n} / n_{\mathrm{GW}}=0.7$. The ITER design point is indicated by the solid circle.

at high and medium triangularity, consistent with the decrease of the Type I ELM frequency with density.

At high density $\left(n_{\text {ped }}>75 \% n_{\mathrm{GW}}\right)$ in high triangularity plasmas, broadband continuous edge-localized MHD activity is observed in between the (low frequency) ELMs. A comparison of the power input and energy loss by Type I ELMs shows that during the interval between ELMs there must be an additional energy loss channel. Under these conditions, the relative energy loss per ELM, $\Delta W_{\mathrm{ELM}} / W_{\text {ped }}$, decreases together with the ELM frequency, which is contrary to the usual Fishpool scaling [34] in which $\Delta W_{\mathrm{ELM}} / W_{\text {ped }}$ and the ELM frequency are approximately inversely correlated. The exact nature of the mechanism leading to these enhanced losses is a subject of further study. If power and particle exhaust can be taken over by continuous MHD activity rather than via an ELM burst, it could possibly be used to advantage for an operational scenario compatible with the first wall.

The various observations on ELM behaviour with pellet injection, impurity seeding, high and medium triangularity discharges with Type I ELMs can be summarized in a unique relationship between the relative energy loss per ELM $\left(\Delta W_{\mathrm{ELM}} / W_{\text {ped }}\right)$ and the collisionality in the pedestal before the ELM burst, $v_{\text {ped }}^{*}$, showing a decrease of the relative ELM losses as a function of $v_{\text {ped }}^{*}$ [35] (figure 10). However, this experimental fact could also be attributed to the dependence of MHD on pedestal collisionality or to other processes 


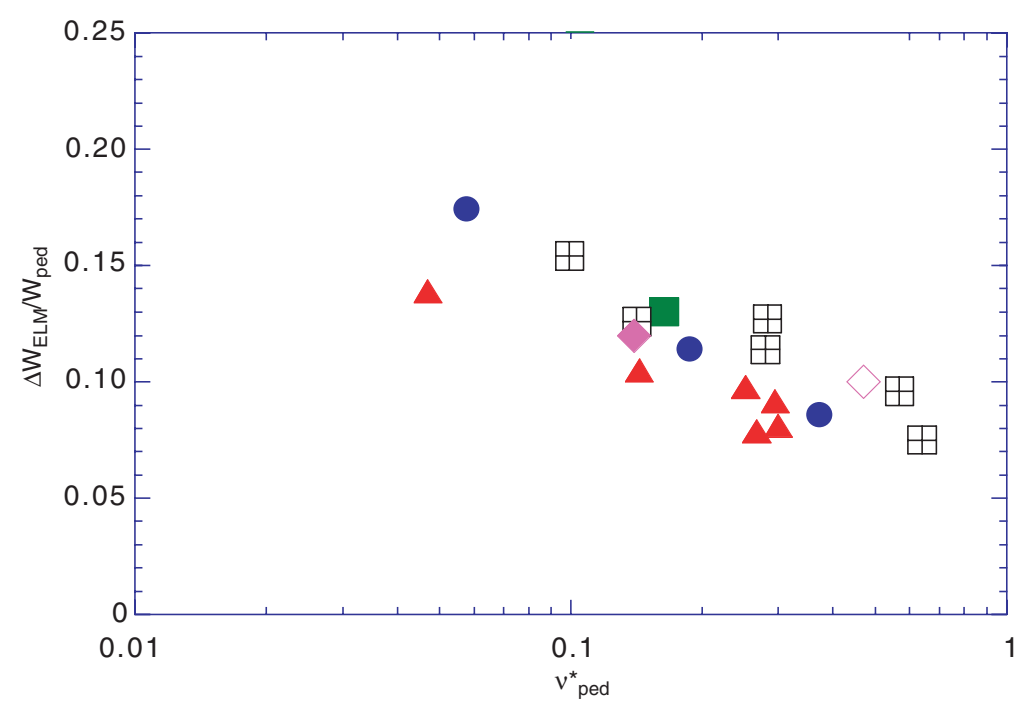

Figure 10. Relative ELM losses $\Delta W_{\mathrm{ELM}} / W_{\text {ped }}$ plotted versus edge pedestal collisionality $v_{\mathrm{ped}}^{*}$, for several discharges in JET (circles: medium triangularity discharges $\delta \sim 0.33$ at $2.5 \mathrm{MA} / 2.7 \mathrm{~T}$; squares: medium triangularity discharges $\delta \sim 0.33$ at $2.5 \mathrm{MA} / 2.7 \mathrm{~T}$ with pellet injection; crossed squares: high triangularity $\delta \sim 0.5$ at $2.5 \mathrm{MA} / 2.7 \mathrm{~T}$; up triangles: medium triangularity discharges $\delta \sim 0.33$ at $1.9 \mathrm{MA} / 2.0 \mathrm{~T}$; full diamonds: low triangularity $\delta \sim 0.2 \mathrm{Ar}$ seeded discharges at $2.5 \mathrm{MA} / 2.4 \mathrm{~T}$; open diamonds: high triangularity $\delta \sim 0.5 \mathrm{Ar}$ seeded discharges at $2.5 \mathrm{MA} / 2.7 \mathrm{~T})$.

associated with the relative duration of the phase of enhanced MHD activity and the characteristic time of the parallel energy loss, depending in different ways on collisionality. According to the exact mechanism, the extrapolation of these results to ITER can differ substantially.

Measurements of power deposition profiles (averaged over ELMs) on the divertor target plates in discharges at $2.5 \mathrm{MA} / 2.4 \mathrm{~T}$ in JET and heated with $12 \mathrm{MW}$ of NBI show that the peak heat flux was strongly reduced (from 20 to $4 \mathrm{MW} \mathrm{m}^{-2}$ ) in discharges at higher densities obtained with $\mathrm{D}_{2}$ puff [36]. This reduction in the peak power load is observed because of the disappearance of the so-called 'narrow feature' in the power load profile on the target [35], indicating a decrease of the fast ion losses from the edge plasma, and is possibly further enhanced by a decrease of the peak power load during the ELM.

Summarizing, medium and high triangularity high density, high confinement plasmas without impurity seeding and with Type I ELMs show the following interesting ELM properties: (i) an ELM frequency which remains low even at high gas fuelling rates, (ii) the relative losses per ELM $\Delta W_{\mathrm{ELM}} / W_{\text {dia }}$ which do not increase with decreasing ELM frequency, and (iii) an ELM average power flux which decreases with increasing fuelling.

In impurity seeded discharges, the presence of the impurity not only increases radiation, but also changes the character of the ELMs [18,34]. In low triangularity discharges, a difference has been observed in the effect of the impurity on the character of the ELMs both during the $\mathrm{D}_{2}$ puff phase and during the afterpuff. Impurity seeding (by Ar or Ne) applied during the main $\mathrm{D}_{2}$ puff phase can change the ELMs from Type I to more benign high frequency Type III ELMs, but with reduced confinement. Impurity seeding applied in the afterpuff reduces the frequency of Type I ELMs and can, with excessive Ar seeding, lead to intermittent ELM-free phases and a tendency for impurity accumulation [13]. 
In high triangularity discharges with adequate impurity seeding not only the ELMs of Type I remain, but they are also reduced in frequency. Also in this case, $\Delta W_{\mathrm{ELM}} / W_{\text {dia }}$ does not follow the usual Fishpool scaling [36] at low frequency, and is reduced by a factor of about 5 [34]. This is consistent with the infrared thermographic measurements, indicating a dramatic drop in the peak and base level temperature in the inner and even more in the outer divertor (figure $5(b)$ ). Although the estimates of ELM power loss from the profiles indicate values on the order of $2.5 \%$ of the stored energy [18], it is still unresolved where, and in what way, the lost power arrives at the first wall. Part of it could well be radiated away before arriving at the target plates where the plasma is possibly in a partial detachment state. However, the extrapolation of this ELM buffering effect has to be further confirmed experimentally, as modelling [28] seems to indicate that with increasing $\Delta W_{\mathrm{ELM}}$ the mitigation effect by radiation could be less efficient. These questions will be addressed in future campaigns. In any case, the results obtained until now show that impurity seeding is an important tool to influence the ELM frequency and amplitude independent of the triangularity of the plasma [34].

\section{MHD in high density, high confinement plasmas}

NTMs often limit the performance of high density, high confinement discharges on JET. Dedicated experiments have demonstrated that NTMs are metastable in standard $q_{95} \sim 3.4$ scenarios as soon as the discharge is in H-mode. Furthermore, at the crash of long sawtooth periods, NTMs can be destabilized at low $\beta_{\mathrm{N}}$ values. On the contrary, reducing the amplitude of the sawteeth reduces the chance to destabilize NTMs, leading to higher $\beta_{\mathrm{N}}$ values before the mode sets in. Therefore specific scenarios have been developed to avoid plasma perturbations (sawteeth with a large sawtooth period, large first ELMs or pellets) which could destabilize NTMs. This has especially been beneficial for the high triangularity, high confinement studies $[8,9]$, and also for the experiments with density peaking on long time scales [25]. The role and the need for a control of large sawteeth in inducing NTMs in discharges has been shown in [37], in particular, in relation to discharges with ICRH.

$3 / 2$ modes deteriorate the performance of standard scenarios, and $2 / 1$ modes lead to a disruption. The higher $m / n=5 / 4$ and $4 / 3$ modes are usually destabilized before the $3 / 2$ modes and have therefore a lower threshold or the island needed to trigger these modes is more easily generated. They are not as detrimental as the $3 / 2$ modes as they are located more towards the plasma centre. However, for the experiments with density peaking, which are more prone to NTMs due to the steep density profiles leading to a non-negligible bootstrap current fraction of $16-20 \%$, they are important as they stop the monotonic increase of the central density.

\section{Conclusions and future work}

Over the last year plasmas have been realized in JET with high confinement $H_{98(y, 2)} \sim 1$ at high densities $\bar{n} / n_{\mathrm{GW}} \sim 1$. This has been possible (i) by increasing the average triangularity of the plasmas, up to $\delta \sim 0.5$, and has been extended to plasmas with medium triangularity $\delta \sim 0.3-0.4$ by increasing the heating power so as to sustain Type I ELMs, (ii) in low and high triangularity plasmas using impurity seeding, showing, along with the good confinement properties, high radiated power fractions (up to 70\%) and (iii) by plasma fuelling with an optimized pellet timing sequence, allowing sufficient time between the successive pellets for the plasma energy to recover without losing too much density. Furthermore, experiments 


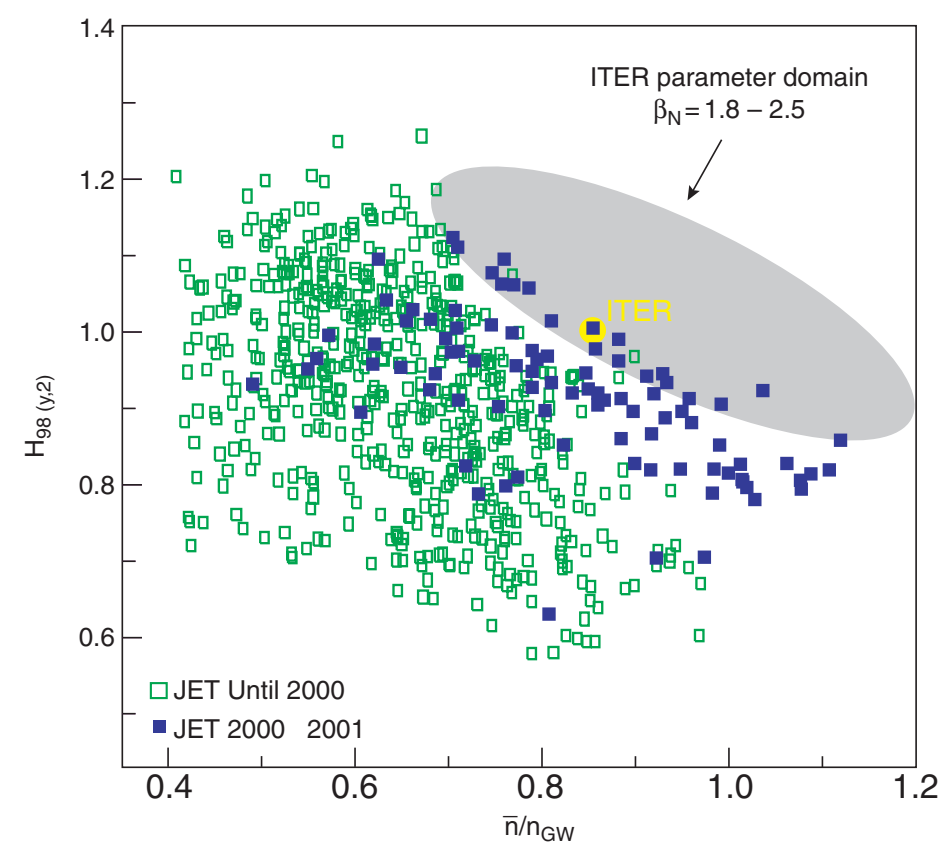

Figure 11. Confinement $H_{98(y, 2)}$ plotted versus the Greenwald factor $\bar{n} / n_{\mathrm{GW}}$ for JET ELMy $\mathrm{H}$-mode discharges since 1994 with $1.8 \mathrm{MA}<I_{\mathrm{p}}<2.7 \mathrm{MA}$. Data are from the JET steady-state database. Open symbols correspond to data obtained before 2000, solid symbols to those from 2000-2001. The full circle corresponds to the ITER working point. The shaded zone roughly corresponds to the ITER parameter domain with $1.8<\beta_{\mathrm{N}}<2.5$.

showed (i) the importance of central heating to maintain sawtooth MHD, preventing impurity accumulation, (ii) the influence of the deuterium gas puff rate in obtaining high confinement and density peaking, and (iii) the importance of Ar seeding to create a radiating belt and to contribute to the mitigation of ELMs.

These techniques allowed JET (figure 11) to reach the ITER $Q=10$ requirements. Moreover, two factors can lead to the decrease of the power load on the target plates: impurity seeding and high triangularity plasmas with broadband inter-ELM MHD activity.

The insights gained, the techniques learned and the data obtained this year are important steps towards ITER. Nevertheless, further work is needed to understand the physics of ELMs at high density and with impurity seeding, and from there to improve the mitigation of ELMs, to extend the stationarity of the plasmas, study the influence of the different heating methods on confinement and ELM behaviour, to mention but a few. In addition, an extension of the results obtained to higher currents, fields and heating powers is necessary to improve the extrapolation of the different high performance regimes to ITER.

\section{Acknowledgments}

It was a great pleasure to share the work with all the European and non-European colleagues at JET this year. The excellent work done by colleagues from UKAEA responsible for the operation of JET has been an important factor in the success of the 2000-2001 experimental campaigns. The work described in this paper has been performed under EFDA (European Fusion Development Agreement). 


\section{References}

[1] Greenwald M et al 1988 Nucl. Fusion 282199

[2] ITER Physics Basis 1999 Nucl. Fusion 392175

[3] Stober J et al 2000 Plasma Phys. Control. Fusion 42 A211

W Suttrop et al 2000 Plasma Phys. Control. Fusion 42 A97

[4] Osborne T H et al 2000 Plasma Phys. Control. Fusion 42 A175

[5] Saibene G, Horton L D, Sartori R et al 1999 Nucl. Fusion 391133

[6] Lomas P J et al 2000 Plasma Phys. Control. Fusion 42 B115

[7] Kamada Y et al 1997 Proc. 16th Conf. Fusion Energy (Montreal, 1996) vol 1 (Vienna: IAEA) p 247

[8] Sartori R 2001 Proc. 28th EPS Conf. on Controlled Fusion and Plasma physics (Funchal, 2001) (Europhys. Conf. Abstracts) vol 25J (Geneva: EPS) P3.003

[9] Saibene G 2001 Proc. 28th EPS Conf. on Controlled Fusion and Plasma physics (Funchal, 2001) (Europhys. Conf. Abstracts) vol 25J (Geneva: EPS) P3.002

[10] Ongena J et al 2001 Plasma Phys. 82188

[11] Strachan J D et al 2000 Plasma Phys. Control. Fusion 42 A81

[12] Dumortier P 2001 Proc. 28th EPS Conf. on Controlled Fusion and Plasma physics (Funchal, 2001) (Europhys. Conf. Abstracts) vol 25J (Geneva: EPS) P3.004

[13] Puiatti M E 2001 Proc. 28th EPS Conf. on Controlled Fusion and Plasma physics (Funchal, 2001) (Europhys. Conf. Abstracts) vol 25J (Geneva: EPS)P3.007

[14] Nave M 2001 Proc. 28th EPS Conf. on Controlled Fusion and Plasma physics (Funchal, 2001) (Europhys. Conf. Abstracts) vol 25J (Geneva: EPS) P3.009

[15] Koslowski H R 2001 Proc. 28th EPS Conf. on Controlled Fusion and Plasma physics (Funchal, 2001)(Europhys. Conf. Abstracts) vol 25J (Geneva: EPS) P3.010

[16] Jackson G 2001 Proc. 28th EPS Conf. on Controlled Fusion and Plasma physics (Funchal, 2001) (Europhys. Conf. Abstracts) vol 25J (Geneva: EPS) P3.017

[17] Tokar' M 2001 Proc. 28th EPS Conf. on Controlled Fusion and Plasma physics (Funchal, 2001) (Europhys. Conf. Abstracts) vol 25J (Geneva: EPS) P3.032

[18] Jachmich S 2001 Proc. 28th EPS Conf. on Controlled Fusion and Plasma physics (Funchal, 2001) (Europhys. Conf. Abstracts) vol 25J (Geneva: EPS) P3.013

[19] Horton L et al Proc. 26th EPS Conf. on Control. Fusion and Plasma Phys. (Maastricht, 1999) paper P1.021

[20] Ehrenberg J et al 1996 Wall effects on particle recycling in tokamaks Physical Processes of the Interaction of Fusion Plasmas with Solids (New York: Academic Press)

[21] Ongena J Evrard M and Mccune D 2000 Fusion Tech. 37313

[22] Eich Th 2001 Proc. 28th EPS Conf. on Controlled Fusion and Plasma physics (Funchal, 2001) (Europhys. Conf. Abstracts) vol 25J (Geneva: EPS) P5.010

[23] Mahdavi M A et al Proc. 18th IAEA Fusion Energy Conf. (Sorrento, Italy, 4-10 Oct. 2000) paper IAEA-CN-77/ EXP1/04

[24] Stober J 8th European Fusion Physics Workshop (Leysin, 13-15 Dec. 2000)

[25] Valovic M 2001 Proc. 28th EPS Conf. on Controlled Fusion and Plasma physics (Funchal, 2001) (Europhys. Conf. Abstracts) vol 25J (Geneva: EPS)P3.008

[26] Lang P T 2001 Proc. 28th EPS Conf. on Controlled Fusion and Plasma physics (Funchal, 2001) (Europhys. Conf. Abstracts) vol 25J (Geneva: EPS) P3.012

[27] Cordey Get al 2001 Proc. 28th EPS Conf. on Controlled Fusion and Plasma physics (Funchal, 2001) (Europhys. Conf. Abstracts) vol 25J (Geneva: EPS) P3.011

[28] Loarte A Proc. 18th IAEA Fusion Energy Conf. (Sorrento, Italy, 4-10 Oct. 2000) paper IAEA-CN-77/ ITERP/11(R)

[29] Kamada Y et al 2000 Plasma Phys. Control. Fusion 42 A247

[30] Stober J et al 2001 Nucl. Fusion 411123

[31] Greenwald M et al 1999 Phys. Plasmas 61943

[32] Parail V 2001 Proc. 28th EPS Conf. on Controlled Fusion and Plasma physics (Funchal, 2001) (Europhys. Conf. Abstracts) vol 25J (Geneva: EPS) P5.027

[33] Hender T, UKAEA Fusion, Association 'UKAEA-Euratom' Private communication

[34] Fishpool G M 1998 Nucl. Fusion 381373

[35] Loarte A 2001 Proc. 28th EPS Conf. on Controlled Fusion and Plasma physics (Funchal, 2001) (Europhys. Conf. Abstracts) vol 25J (Geneva: EPS) P3.005 
[36] Fundamenski W et al 2001 Proc. 28th EPS Conf. on Controlled Fusion and Plasma physics (Funchal, 2001) (Europhys. Conf. Abstracts) vol 25J (Geneva: EPS) P4.073

[37] Sauter O et al 2001 Proc. 28th EPS Conf. on Controlled Fusion and Plasma physics (Funchal, 2001) (Europhys. Conf. Abstracts) vol 25J (Geneva: EPS) P5.001

[38] Jackson G 2001 Proc. 28th EPS Conf. on Controlled Fusion and Plasma physics (Funchal, 2001) (Europhys. Conf. Abstracts) vol 25J (Geneva: EPS) P3.017 